\title{
Dynamics study of nonlinear mechanical vibration systems
}

\author{
Pang Junru \\ China Agricultural University, Beijing, China, 100083
}

Keywords: nonlinear; mechanical vibration system; disc brake; dynamic analysis

\begin{abstract}
Nowadays, the structure of modern machinery is increasingly complex, the speed of the machine continues to increase, so the problem of nonlinear vibration caused by gap and dry friction is becoming more and more common. In this paper, a dynamic model of a common mechanical system is established. The motion law of the system under different conditions is studied by combining theoretical calculation with numerical simulation. Taking the interaction between the brake pad and the brake disc as an example, based on the analysis of vertical excitation and frictional flutter, the dynamic characteristics of the nonlinear mechanical vibration system are deeply studied. It provides a reference for the improvement of bad vibration and noise, and make the train stable and comfortable.
\end{abstract}

\section{Introduction}

As an approximation of nonlinear systems, linear systems are more easier to be solved, and the solution of the system satisfies the superposition principle. It has the characteristics of predictability and certainty, which makes it be favored by researchers in the past. However, the actual natural phenomena and social phenomena are mostly complex and changeable, most of which always obey the nonlinear law. The internal motion of the nonlinear system often shows strong randomness. In order to study the variation of various state variables, nonlinear dynamics is applied.

2.At present, the research on the periodic motion of the collision-vibration system with only gaps is relatively mature, and dry friction as a dissipative force can also bring about unavoidable noise hazards while achieving the purpose of mechanical system damping ${ }^{[1]}$. It is very difficult to solve the response of the system by analytical method, but it has important research value and provides a chance by integrating the two nonlinear factors in the course of the research ${ }^{[2-3]}$. Many scholars have carried out a lot of research on nonlinear mechanical vibration systems, but these mathematical modeling are idealized, and difficult to be applied in engineering practice ${ }^{[4-5]}$. The studies on high-dimensional collisional vibration systems for dry friction and gap coupling are very few. It has great blindness ${ }^{[6-7]}$. Therefore, this paper will study a simplified dynamic model of a two-degree-of-freedom vehicle disc brake system with clearance and dry friction.

\section{Dynamic analysis of disc brake system with two degrees of freedom}

\subsection{Overview of engineering background}

As an auxiliary braking system, disc braking plays the role of emergency deceleration or braking when the vehicle fails in other main braking modes. Of course, any brake system has certain limitations, disc brake system is no exception, for example, after the use of disc brake, the wheel tread has not been scratched for a long time, resulting in increased adhesion between the wheel and the track, affecting the safety of driving; brake disc and brake disc friction will bring vehicle flutter and noise; due to installation The brake disc causes the spring weight and impact vibration to increase, consuming a part of the traction power and other adverse effects. Therefore, the analysis of the dynamic performance of the disc brake system has a positive practical significance for the continuous optimization of the vehicle braking performance. 


\subsection{Mechanics model}

\subsubsection{Dynamic model}

When the disc brake system works, the pressure generated by the brake cylinder will be made of synthetic materials, two brake pads pressed tightly on both sides of the disc, through energy conversion to achieve the purpose of braking. Because the wheel set is constantly excited by the track, the vehicle inevitably produces vertical vibration, and the brake disc installed on the axle will also produce vibration, and the brake disc and the brake disc are fixed on the frame through a certain connection, so the brake disc also has certain vibration.

As shown in Figure 1, this paper simplifies the disc brake system into a two-degree-of-freedom dynamic model with clearance and dry friction, where M1 represents the mass of the brake disc, K1 represents the stiffness coefficient of the brake disc, and C1 represents the damping coefficient of the brake disc. M1 is connected to the left fixed end through K1 and C1; M2 represents the mass of the brake disc, K2 represents the stiffness coefficient of the brake disc, and C2 represents the damping coefficient of the brake disc, M2 is connected to the right side of M1 through K2 and C2, which is subjected to the normal brake pressure $\mathrm{fn}$. It is assumed that the two masses are only vibrate in the horizontal direction and are respectively driven by harmonic excitation $P_{i} \sin (\Omega \mathrm{T}+\tau)(i=1,2)$. The coordinate system is established at the equilibrium position, which make the distance between M1 and M2 is D. They will collide each other when the displacement of M1 and M2 satisfies the condition: X2 - X1 = D. The damping used in the mechanical model is Rayleigh type damping, and the recovery coefficient $\mathrm{R}$ is used to determine the collision process.

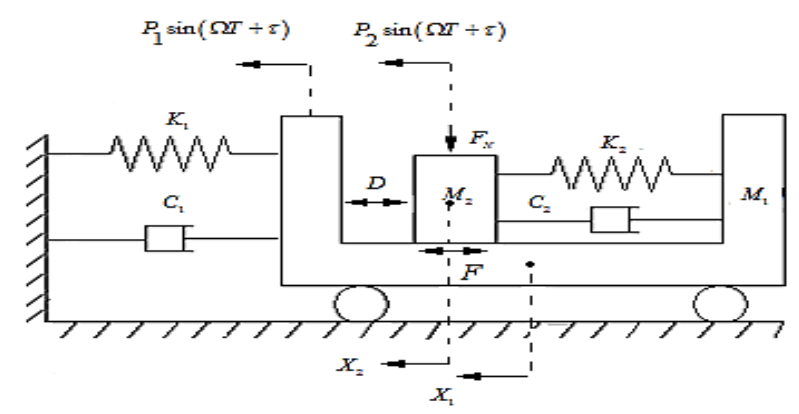

Figure 1. Simplified model of a two-degree-of-freedom disc brake system

\subsubsection{Friction model}

Many kinds of friction models have been proposed in mechanical systems so far. This paper selects the coulomb model in the static friction model, the most commonly used discontinuous friction model in the damping system. In this model, the frictional force is proportional to the normal load, and the direction is opposite to the moving direction. It is assumed that the static friction coefficient and the dynamic friction coefficient of the friction force are equal and the dynamic friction coefficient is independent of the relative velocity of the two contact faces, and the dynamic friction coefficient is considered to be a constant. This ideal dry friction model is simple in structure and can explain the adhesion-sliding characteristics of the system, making it easy to analyze the nonlinear characteristics of the impact vibration system. The expression of the Coulomb model is:

$$
f(v)=f_{c} \operatorname{sgn}(v)
$$

where $\mathrm{f}$ is the frictional force, $\mathrm{v}$ is the relative sliding speed, $f_{c}=\mu\left|f_{n}\right|$ represents the coulomb friction, and $\mu$ represents the friction coefficient, $f_{n}$ represents the normal load, and $\operatorname{sgn}(v)$ represents the sign function.

\subsection{Motion differential equations and its periodic solutions}

\subsubsection{Differential equations and periodic solutions of sliding state}

According to Newton's second law, the differential equation between the two adjacent collisions 
of the system shown in Figure 1 is as follows:

$$
\begin{gathered}
{\left[\begin{array}{cc}
M_{1} & 0 \\
0 & M_{2}
\end{array}\right]\left\{\begin{array}{l}
\ddot{X}_{1} \\
\ddot{X}_{2}
\end{array}\right\}+\left[\begin{array}{cc}
C_{1}+C_{2} & -C_{2} \\
-C_{2} & C_{2}
\end{array}\right]\left\{\begin{array}{l}
\dot{X}_{1} \\
\dot{X}_{2}
\end{array}\right\}+\left[\begin{array}{cc}
K_{1}+K_{2} & -K_{2} \\
-K_{2} & K_{2}
\end{array}\right]\left\{\begin{array}{l}
X_{1} \\
X_{2}
\end{array}\right\}+F=\left\{\begin{array}{l}
P_{1} \\
P_{2}
\end{array}\right\} \sin (\Omega \mathrm{T}+\tau)} \\
X_{2}-X_{1}<D
\end{gathered}
$$

In order to make the calculation process simpler, it is dimensionlessly quantized and decoupled. Similar to the decoupling method of the three-degree-of-freedom system, the tedious derivation process is omitted here, and the analytical solution of the response when the system is in the sliding state is directly given as follow:

$$
x_{i}(t)=\sum_{j=1}^{2} \psi_{i j}\left(e^{-\eta_{j} t}\left(a_{j} \cos \omega_{d j} t+b_{j} \sin \omega_{d j} t\right)+A_{j} \sin (\omega t+\tau)+B_{j} \cos (\omega t+\tau)\right)
$$

\subsubsection{Differential equations and periodic solutions of the stuck state}

When the vibrator M2 sticks to M1, the whole system becomes a single degree of freedom system. At this time, the speeds of M2 and M1 satisfy the relationship $\dot{x}_{1}(0)=\dot{x}_{2}(0)$, that is, their relative speed is 0 , and the solution of the static friction force fs can be solved as follow :

$$
f_{s}=\left(F_{1}-F_{2}\right) \sin (\omega t+\tau)-2 \zeta\left(\omega_{n 1}-\omega_{n 2}\right) \cdot \dot{x}_{2}(0)+\frac{\sqrt{\left(1+\mu_{m}+\mu_{k}\right)^{2}-4 \mu_{m} \mu_{k}}}{\mu_{m}} \cdot x_{2}(0)
$$

So the conditions when the system is stuck are:

$$
\left\{\begin{array}{l}
\dot{x}_{1}(0)=\dot{x}_{2}(0) \\
f_{s}=\left(F_{1}-F_{2}\right) \sin (\omega t+\tau)-2 \zeta\left(\omega_{n 1}-\omega_{n 2}\right) \cdot \dot{x}_{2}(0)+\frac{\sqrt{\left(1+\mu_{m}+\mu_{k}\right)^{2}-4 \mu_{m} \mu_{k}}}{\mu_{m}} \cdot x_{2}(0)
\end{array}\right.
$$

where $f_{s} \in\left[-f_{\max }, f_{\max }\right], f_{\max }=\mu_{0} N, f_{\max }$ indicates the maximum static friction, $\mu_{0}$ is the maximum static friction factor, and $\mathrm{N}$ is the positive pressure.

When the system is in a stuck state, the system is a single degree of freedom system, and its differential equation of motion can be write as :

$$
\left(M_{1}+M_{2}\right) X_{1}+C_{1} X_{1}+K_{1} X_{1}=p_{1} \sin (\Omega \mathrm{T}+\tau)
$$

The analytical solution of the differential equation when the system is in the stuck state is:

$$
\begin{gathered}
x(t)=e^{-\zeta \omega_{n} t}\left(a \cos \left(\omega_{d} t\right)+b \sin \left(\omega_{d} t\right)\right)+X \sin (\omega t-\varphi) \\
\dot{x}(t)=a e^{-\zeta \omega_{n} t}\left(-\zeta \omega_{n} \cos \omega_{d} t-\omega_{d} \sin \omega_{d} t\right)+b e^{-\zeta \omega_{n} t}\left(\omega_{d} \cos \omega_{d} t-\zeta \omega_{n} \sin \omega_{d} t\right)+X \omega \cos (\omega t-\varphi)
\end{gathered}
$$

\subsection{Numerical Simulation Analysis}

\subsubsection{Analysis of the influence of friction on system response}

Select a set of dimensionless parameters: $\mu_{m}=2, \mu_{k}=2, \mu_{c}=0.2$, $\zeta=0.00031, f_{20}=0.5, R=0.7, d=0.35, \omega=0.577185$. Keeping the other parameters unchanged, when the friction force $\mathrm{f}=0.32$, due to the existence of damping, the system energy is continuously consumed, the response exhibits quasi-periodic motion, but the damping is small, and the response amplitude has a slight attenuation. The vibrator M1 briefly stays in the equilibrium position, and then continues to do symmetric motion; as $\mathrm{f}$ increases, the symmetry of M1 is destroyed. When $\mathrm{f}=1.36$, the amplitude attenuation of $\mathrm{M} 1$ is aggravated, the retention disappears, and only along the equilibrium position. One side of the motion, as shown in Figure 2; when $f=2.21$, the system vibration trend is exactly the opposite of $f=0.32$, the vibrator only moves along the side of the equilibrium position. Overall, as the dry friction of the system increases, the amplitude of the quasi-periodic motion increases continuously, but the dry friction has no significant effect on the system's motion trend. 

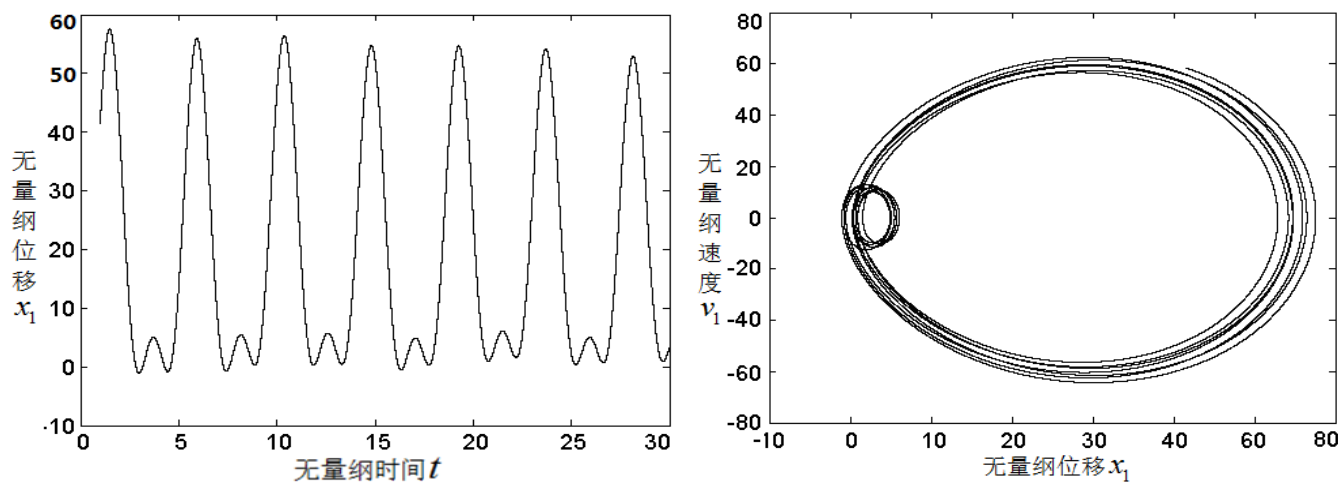

Fig. 2 Simulation analysis of the quasi-periodic motion of the system with dry friction $(f=1.36)$

\subsubsection{Dynamics analysis of system resonance}

Select a set of dimensionless parameters of the system: $\mu_{m}=2, \mu_{k}=1$, $\mu_{c}=0.2, \zeta=0.041, f_{20}=0.5 R=0.7, d=0.13, f=0.32$. The transient response of the system is severe under vibration, and finally the attenuation is stable to a certain amplitude for simple harmonic vibration. When $\omega=1$, the vibrator M1 satisfied $\omega_{n 1}=\omega$, the resonance will occurs and the amplitude reaches the maximum. The limit cycle occurs in the phase plane. When it is less than or greater than the frequency at which the system resonates, the response is weaker than the response of the system at resonance, and as $\mathrm{w}$ increases in a certain interval, the attenuation of the system vibration is intensified, and the limit cycle on the phase diagram is also constantly Zoom out, the process of change is shown in Figure 3.
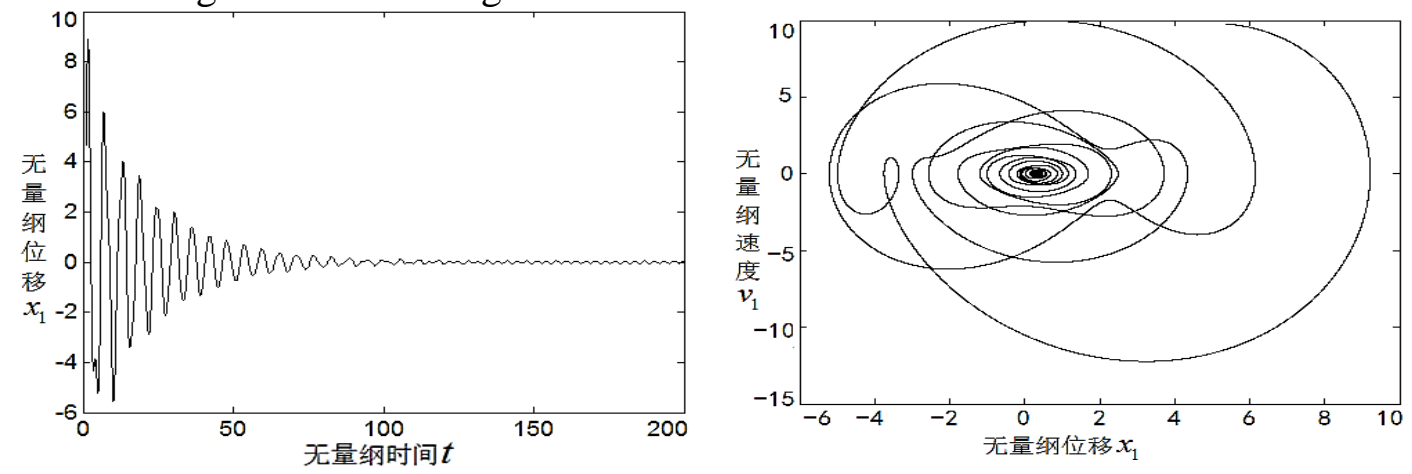

Figure 3 Simulation analysis of the resonant and non-resonant states $(\mathrm{w}=1.926)$

\subsubsection{Influence of initial braking speed on system vibration performance}

Take a set of dimensionless parameters of the system: $\mu_{m}=3.3, \mu_{k}=2.3$, $\mu_{c}=0.6, \zeta=0.69, f_{20}=0.001, R=0.7, d=0.2, f=0.32, \omega=1.086685$. When $v_{1}(0)=1.52$, the initial vibration of the brake disc and the brake pad is disordered and the amplitude is large, then the vibration gradually decays and converges to a stable period 1 motion, the vibration of the brake disc It is stronger than the brake pad, as shown in Figure 4; on the phase diagram the system trajectory is eventually attracted around the limit ring. Repeated simulations show that when the initial braking speed is greater than a certain critical speed, as the braking speed increases, the amplitude of the system vibration increases at the beginning of braking, but quickly returns to a stable state.

When $v_{1}(0)=0.15$, the amplitude of the brake pad and the brake disc is significantly increased compared to the amplitude of $v_{1}(0)=0.26$. The simulation finds that when the braking speed is less than a certain critical speed, the braking system vibrates in a certain speed range. The amplitude increases with the decrease of the braking speed. In order to improve the running stability of the train, this braking speed range should be avoided as much as possible. 

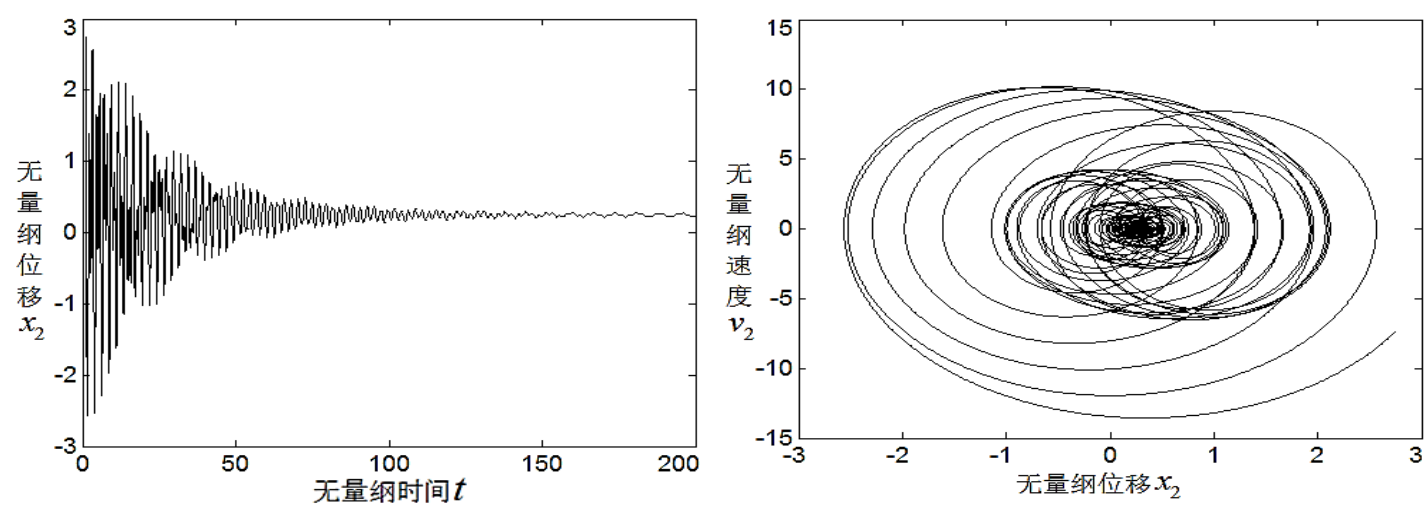

Figure 4 Simulation results at braking speed $v(0)=1.52$

\subsection{Analysis of simulation results}

We obtain the following conclusions: Increasing friction has a great influence on the periodic motion of the system. The vibration is most intense when the system resonates, and the amplitude is very dependent on the damping. When the braking speed is greater than the critical braking speed when the system flutters, the amplitude of the vibration increases with the increase of the braking speed. The excitation amplitude increases continuously in a certain interval, and the vertical vibration amplitude of the brake disc and the brake pad is also larger, but the variation of the excitation amplitude has little influence on the amplitude and frequency of the flutter. The excitation frequency increases continuously in a certain interval, the frictional flutter becomes weaker and weaker, and finally disappears. The system only makes vertical vibration, and the amplitude of vertical vibration has a tendency to increase with the increase of excitation frequency. The stability of the train has a great influence.

\section{Conclusion}

In this paper, the dynamics of mechanical vibration systems with nonlinear factors are studied. A two-degree-of-freedom disc brake system considering the two nonlinear factors of clearance and dry friction is studied. The critical conditions for the adhesion-sliding-collision motion of the system are given. The differential equations of different motion states of the system are derived by semi-analytical method. The numerical simulation is used to analyze the damping ratio of dry friction and resonance through numerical iterative programming. The influence of the parameters such as the initial braking speed on the braking performance of the system under different stress conditions. Through the simulation analysis of the vibration law of the vehicle disc brake system under different conditions, it provides some reference basis for reducing the vibration and noise brought by friction braking and improving the stability and comfort of the train.

\section{References}

[1] LeDong Zhu, XiaoLiang Meng, LinQing Du, MingChang Ding. A Simplified Nonlinear Model of Vertical Vortex-Induced Force on Box Decks for Predicting Stable Amplitudes of Vortex-Induced Vibrations[J]. Engineering.2017,(6): 854-862.

[2] M. Faraji Oskouie, R. Ansari, F.Sadeghi.Nonlinear vibration analysis of fractional viscoelastic Euler-Bernoulli nanobeams based on the surface stress theory[J].Acta Mechanica Solida Sinica.2017,(4): 416-424.

[3] Yangyang Luo, Min Tang, Qiao Ni, Yikun Wang, Lin Wang.Nonlinear Vibration of A Loosely Supported Curved PipeConveying Pulsating Fluid under Principal Parametric Resonance[J].Acta Mechanica Solida Sinica.2016,(5):468-478.

[4] Neild S A, Champneys A R, Wagg D J, et al. The use of normal forms for analysing nonlinear mechanical vibrations[J]. 2015, 373(2051). 
[5] Kovacic I, Radomirovic D. Mechanical Vibrations: Fundamentals with Solved Examples[M]. 2017.

[6] Sira-Ramírez H, Llanes-Santiago O. Sliding Mode Control of Nonlinear Mechanical Vibrations[J]. Journal of Dynamic Systems Measurement \& Control, 2000, 122(4):674-678.

[7] Wu Y, Badel A, Formosa F, et al. Nonlinear vibration energy harvesting device integrating mechanical stoppers used as synchronous mechanical switches[J]. Journal of Intelligent Material Systems \& Structures, 2016, 25(14):1658-1663.

[8] Cao D X, Leadenham S, Erturk A. Internal resonance for nonlinear vibration energy harvesting[J]. European Physical Journal Special Topics, 2015, 224(14-15):2867-2880.

[9] Raoul Saggini; Emilio Ancona. The Applied Mechanical Vibration as Whole-body and Focal Vibration[J].The Mechanical Vibration: Therapeutic Effects and Applications.2017:25-88.

[10] Iosifil. Vulfson. Parametric Vibrations Excitation in Cyclic Mechanisms[J].Advances in Mechanical Engineering.2017:133-143. 